\title{
Non-minimally coupled Dirac equation with torsion: Poincaré gauge theory of gravity with even and odd parity terms
}

\author{
Muzaffer Adak \\ Department of Physics, Faculty of Arts and Sciences, \\ Pamukkale University, 20017 Denizli, Turkey \\ madak@pau.edu.tr \\ (Dated: 13 March 2012, file DiracTorsion11g.tex)
}

\begin{abstract}
We take a Dirac field non-minimally coupled to the gravitational field within the framework of the Poincaré gauge theory of gravity with torsion and curvature. We study the subcase of "weak" gravity, that is, the gravitational Lagrangian depends only linearly on the curvature and quadratically on the torsion. We include all pieces in curvature and torsion that are of odd parity. The second field equation of gravity is derived by varying the Lorentz connection. We solve it with respect to the torsion and decompose the first field equation of gravity and the Dirac equation into Einsteinian pieces and post-Riemannian terms.
\end{abstract}

PACS numbers: 04.50.Kd, 03.65.Pm, 11.15.-q 


\section{INTRODUCTION}

When parity violation in quantum gravity is addressed, one generally studies the coupling of fermionic degrees of freedom in the presence of torsion. This is done by reading out the most relevant terms in the low-energy effective lagrangian. In the Poincare gauge theory of gravity the dynamical variables are given by orthonormal coframe $\vartheta^{\alpha}$ and the Lorentz connection $\Gamma^{\alpha \beta}$. If one applies the effective field theory point of view to gravity in the low-energy limit, then to leading order the low-energy effective lagrangian contains exactly six terms, see e.g. [1] and references therein. These are the Euler term $R^{\alpha \beta} \wedge R^{\gamma \delta} \epsilon_{\alpha \beta \gamma \delta}$, the Pontryagin term $R^{\alpha \beta} \wedge R_{\alpha \beta}$, the Nieh-Yan term $T_{\alpha} \wedge T^{\alpha}+R_{\alpha \beta} \wedge \vartheta^{\alpha \beta}$, the Einstein-Hilbert term $R_{\alpha \beta} \wedge \eta^{\alpha \beta}$, the cosmological term $\lambda_{0} \eta$ and the curvature pseudoscalar $R_{\alpha \beta} \wedge \vartheta^{\alpha \beta}$. Since the first two of them are topological invariants and the Nieh-Yan term is an exact form, people concentrate on the last three bulk terms as the most general four-dimensional low-energy gravitational lagrangian. When a Dirac field is minimally coupled to that gravitational lagrangian with its three bulk terms, the parity-violating vector-axial interaction is absent and correspondingly parity is not violated by the gravitational field. If the coupling is nonminimal, then one can see that the parity-violating term is proportional to the Immirzi parameter [1], [2]. That most general gravitational lagrangian corresponds to the first three terms of our lagrangian (18). We compare with the literature and find that the coupling coefficient $b_{0}$ is proportional to the inverse of the Immirzi parameter.

The parity preserving $R+R^{2}+T^{2}$-type theory (with or without matter fields) have been studied in the early 1980s by different groups for different aims in the literature. For example, in Ref[3] Hayashi and Shirafuji minimally coupled a Dirac field and analyzed the field equations, in Ref[4] Blagojevic and Nikolic investigated general aspects of the Hamiltonian structure of the Poincare gauge theory of gravity of that type in the time gauge and in Ref[5] Nikolic applied Dirac's Hamiltonian method for constrained systems to that type of gravity interacting with an arbitrary matter field. In a recent paper [6] Baekler et al proposed a cosmological model in the framework of the Poincare gauge theory of gravity of that type with even parity and odd parity.

In general, there are two types of actions: minimal and non-minimal. The non-minimal interactions with spinors and scalars provide certain advantages at the quantum level, for they give the possibility to construct renormalizable theory. For more detailed discussion on 
non-minimal couplings see the review [7]. Our wish here is to investigate the possible parity effects of a non-minimally coupled Dirac field to the most general "weak" gravitational interactions by studying the most general $R+T^{2}$-type gravity lagrangian including all possible even and odd parity terms. As it contains eight parameters $\alpha, \lambda_{0}, a_{0}, b_{0}, k_{1}, k_{2}, k_{3}, \ell_{2}$ in general, the non-minimally Dirac coupled system can be described by at most seven parameters $\left(k_{1}=0\right.$ without loss of generality).

What we do is basically to consider that total lagrangian and to derive the equations of motions via independent variations in accord with the orthonormal coframe, the Lorentz connection 1-forms and the Dirac spinor. Then we solve explicitly the SECOND field equation for the torsion tensor. At the next step we rewrite the remaining field equations naively in terms of the familiar Riemannian terms plus other terms depending on the Dirac field and its current vectors and pseudo-vectors. Finally we give the equivalent lagrangian written in the Riemannian spacetime. This form of the theory should also be useful in view of future experiments that could put constraints on the parameters of the theory, and test possible effects resulting from a non-vanishing torsion. The same strategy was followed in [8] in which the gravity lagrangian contains only three parameters corresponding to our coupling coefficients $a_{0}, b_{0}, \ell_{2}$ and the matter coupling is minimal. Correspondingly the novelty of our work is to display the most general gravity model containing the non-minimal coupled Dirac field and the non-propagating torsion including all eight parameters known from the literature.

\section{MATHEMATICAL PRELIMINARIES}

Spacetime is denoted by the triple $\{M, g, \nabla\}$ where $M$ is differentiable 4-dimensional manifold, $g=g_{i j}(x) d x^{i} \otimes d x^{j}=g_{\alpha \beta} \vartheta^{\alpha}(x) \otimes \vartheta^{\beta}(x)$ is the metric tensor with $g_{\alpha \beta}=\operatorname{diag}(-1,1,1,1)$, and $\nabla$ represents the linear connection. We require that $M$ is orientable, otherwise one has problems with defining the volume form and the Hodge dual. We take the conventions that $\alpha, \beta, \cdots=0,1,2,3$ denote the orthonormal (anholonomic) indices and $i, j, \cdots=\hat{0}, \hat{1}, \hat{2}, \hat{3}$ the coordinate indices. Correspondingly, $\vartheta^{\alpha}(x)=e_{i}{ }^{\alpha}(x) d x^{i}$, where $d x^{i}$ is a coordinate coframe and $e_{i}^{\alpha}(x)$ are tetrad components (or coordinate components of orthonormal coframe). Thus as $e_{\alpha}(x)$ denotes the orthonormal frame, $\partial_{i}$ the coordinate frame such that $e_{\alpha}(x)=e_{\alpha}{ }^{i}(x) \partial_{i}$. 
Frame and coframe are dual to each other,

$$
\left.\vartheta^{\alpha}\left(e_{\beta}\right) \equiv e_{\beta}\right\lrcorner \vartheta^{\alpha}=\delta_{\beta}^{\alpha},
$$

where $\delta_{\beta}^{\alpha}$ is the Kronecker symbol and $\lrcorner$ denotes the interior product. The linear connection is determined by local (linear) connection 1-forms $\Gamma_{\alpha}^{\beta}(x)$ as follows: $\nabla_{e_{\alpha}} e_{\beta}=-\Gamma_{\beta}^{\gamma}\left(e_{\alpha}\right) e_{\gamma}$. We prefer to work with orthonormal frame because of its coordinate independence. It is also necessary when one wants to introduce spinors. With the shorthand notation $\vartheta^{\alpha} \wedge \vartheta^{\beta} \wedge \cdots \equiv$ $\vartheta^{\alpha \beta \cdots}$ and the volume 4 -form $\eta:={ }^{\star} 1$ we use the following eta-basis,

$$
\begin{aligned}
\eta^{\alpha} & \left.:=e^{\alpha}\right\lrcorner \eta={ }^{\star} \vartheta^{\alpha}, \\
\eta^{\alpha \beta} & \left.:=e^{\beta}\right\lrcorner \eta^{\alpha}={ }^{\star} \vartheta^{\alpha \beta}, \\
\eta^{\alpha \beta \gamma} & \left.:=e^{\gamma}\right\lrcorner \eta^{\alpha \beta}={ }^{\star} \vartheta^{\alpha \beta \gamma}, \\
\eta^{\alpha \beta \gamma \rho}: & \left.:=e^{\rho}\right\lrcorner \eta^{\alpha \beta \gamma}={ }^{\star} \vartheta^{\alpha \beta \gamma \rho},
\end{aligned}
$$

where $\star$ denotes Hodge star.

A geometry (in orthonormal frames) is determined by the Cartan structure equations

$$
\begin{aligned}
Q_{\alpha \beta} & :=-D g_{\alpha \beta}=\Gamma_{\alpha \beta}+\Gamma_{\beta \alpha}, \\
T^{\alpha} & :=D \vartheta^{\alpha}=d \vartheta^{\alpha}+\Gamma_{\beta}^{\alpha} \wedge \vartheta^{\beta}, \\
R_{\alpha}^{\beta} & :=D \Gamma_{\alpha}^{\beta}:=d \Gamma_{\alpha}^{\beta}-\Gamma_{\alpha}^{\gamma} \wedge \Gamma_{\gamma}^{\beta},
\end{aligned}
$$

where $Q_{\alpha \beta}$ are the nonmetricity 1-forms, $T^{\alpha}$ the torsion 2-forms and $R_{\alpha}{ }^{\beta}$ the curvature 2-forms. The operators $d$ and $D$ denote the exterior derivative and the covariant exterior derivative, respectively. These tensors satisfy the Bianchi identities

$$
\begin{aligned}
D Q_{\alpha \beta} & =R_{\alpha \beta}+R_{\beta \alpha}, \\
D T^{\alpha} & =R_{\beta}^{\alpha} \wedge \vartheta^{\beta}, \\
D R_{\alpha}{ }^{\beta} & =0 .
\end{aligned}
$$

Differentiating the $\eta$ 's, we find the following useful relations

$$
\begin{aligned}
D \eta_{\alpha} & =-2 Q \wedge \eta_{\alpha}+T^{\epsilon} \wedge \eta_{\alpha \epsilon}, \\
D \eta_{\alpha \beta} & =-2 Q \wedge \eta_{\alpha \beta}+T^{\epsilon} \wedge \eta_{\alpha \beta \epsilon}, \\
D \eta_{\alpha \beta \gamma} & =-2 Q \wedge \eta_{\alpha \beta \gamma}+T^{\epsilon} \wedge \eta_{\alpha \beta \gamma \epsilon}, \\
D \eta_{\alpha \beta \gamma \delta} & =-2 Q \wedge \eta_{\alpha \beta \gamma \delta},
\end{aligned}
$$


where $Q=Q^{\alpha}{ }_{\alpha} / 4$ is the Weyl covector.

In this work we set nonmetricity to zero. This means, in orthonormal frames, $\Gamma_{\alpha \beta}=-\Gamma_{\beta \alpha}$ and $R_{\alpha \beta}=-R_{\beta \alpha}$. That spacetime is known as the Riemann-Cartan spacetime with metric compatible connection. In this case, the linear connection 1-forms can be decomposed as follows [9],[10]:

$$
\Gamma_{\alpha}^{\beta}=\widetilde{\Gamma}_{\alpha}^{\beta}-K_{\alpha}^{\beta}
$$

where the contortion 1-forms $K_{\alpha}{ }^{\beta} \wedge \vartheta_{\beta}=T_{\alpha}$ or

$$
\left.\left.\left.\left.2 K_{\alpha \beta}=e_{\alpha}\right\lrcorner T_{\beta}-e_{\beta}\right\lrcorner T_{\alpha}-\left(e_{\alpha}\right\lrcorner e_{\beta}\right\lrcorner T_{\gamma}\right) \vartheta^{\gamma},
$$

the Christoffel 1-forms (or Riemannian connection 1-forms) $\widetilde{\Gamma}_{\alpha}{ }^{\beta}$

$$
\left.\left.\left.\left.\left.\left.2 \widetilde{\Gamma}_{\alpha \beta}=d g_{\alpha \beta}+\left(e_{\alpha}\right\lrcorner d g_{\beta \gamma}-e_{\beta}\right\lrcorner d g_{\alpha \gamma}\right) \vartheta^{\gamma}+e_{\alpha}\right\lrcorner d \vartheta_{\beta}-e_{\beta}\right\lrcorner d \vartheta_{\alpha}-\left(e_{\alpha}\right\lrcorner e_{\beta}\right\lrcorner d \vartheta_{\gamma}\right) \vartheta^{\gamma} .
$$

In orthonormal frames $\widetilde{\Gamma}_{\alpha \beta}$ turns out to be

$$
\left.\left.\left.\left.2 \widetilde{\Gamma}_{\alpha \beta}=e_{\alpha}\right\lrcorner d \vartheta_{\beta}-e_{\beta}\right\lrcorner d \vartheta_{\alpha}-\left(e_{\alpha}\right\lrcorner e_{\beta}\right\lrcorner d \vartheta_{\gamma}\right) \vartheta^{\gamma} .
$$

In this work riemannian quantities are labeled by a tilde, e.g. Riemann (curvature) 2-form

$$
\widetilde{R}_{\alpha}^{\beta}=d \widetilde{\Gamma}_{\alpha}^{\beta}-\widetilde{\Gamma}_{\alpha}^{\gamma} \wedge \widetilde{\Gamma}_{\gamma}^{\beta}
$$

\section{GRAVITATIONAL LAGRANGIAN}

In the framework of the Poincaré gauge theory, the gravitational lagrangian is composed of parity even and parity odd pieces [6]:

$$
V_{ \pm}=V_{+}+V_{-}
$$

with

$$
V_{+}=\frac{1}{2 \kappa}\left(-a_{0}{ }^{(6)} R^{\alpha \beta} \wedge \eta_{\alpha \beta}-2 \lambda_{0} \eta+T^{\alpha} \wedge \sum_{I=1}^{3} a_{I}^{\star(I)} T_{\alpha}\right)-\frac{1}{2 \varrho}\left(R^{\alpha \beta} \wedge \sum_{I=1}^{6} w_{I}^{\star(I)} R_{\alpha \beta}\right)
$$

and

$$
\begin{aligned}
V_{-}= & -\frac{b_{0}}{2 \kappa}{ }^{(3)} R_{\alpha \beta} \wedge \vartheta^{\alpha \beta}+\frac{1}{\kappa}\left(\sigma_{1}{ }^{(1)} T^{\alpha} \wedge{ }^{(1)} T_{\alpha}+\sigma_{2}{ }^{(2)} T^{\alpha} \wedge{ }^{(3)} T_{\alpha}\right) \\
& -\frac{1}{2 \varrho}\left(\mu_{1}{ }^{(1)} R^{\alpha \beta} \wedge{ }^{(1)} R_{\alpha \beta}+\mu_{2}{ }^{(2)} R^{\alpha \beta} \wedge{ }^{(4)} R_{\alpha \beta}+\mu_{3}{ }^{(3)} R^{\alpha \beta} \wedge{ }^{(6)} R_{\alpha \beta}+\mu_{4}{ }^{(5)} R^{\alpha \beta} \wedge{ }^{(5)} R_{\alpha \beta}\right)
\end{aligned}
$$


where $\kappa$ and $\varrho$ are respectively the weak and strong gravitational constants, $a_{0}, b_{0}, a_{I}, w_{I}, \sigma_{I}, \mu_{I}$ are the dimensionless coupling constants and $\lambda_{0}$ is the cosmological constant, ${ }^{(I)} T^{\alpha}$ are the irreducible decompositions of the torsion and ${ }^{(I)} R_{\alpha \beta}$ are the irreducible pieces of the curvature. This lagrangian obtained from a classic field theoretical view point is equivalent to one [11] obtained from a quantum field theoretical view point, see [12]. If we specialize to the subcase of weak gravity, that is, we drop the terms that are multiplied by $1 / \varrho$, then the lagrangian reads

$$
\begin{aligned}
\left.V_{ \pm}\right|_{\text {weak }}=\frac{1}{2 \kappa}( & -a_{0}{ }^{(6)} R^{\alpha \beta} \wedge \eta_{\alpha \beta}-2 \lambda_{0} \eta-b_{0}{ }^{(3)} R_{\alpha \beta} \wedge \vartheta^{\alpha \beta} \\
& \left.+T^{\alpha} \wedge \sum_{I=1}^{3} a_{I}{ }^{\star(I)} T_{\alpha}+2 \sigma_{1}{ }^{(1)} T^{\alpha} \wedge{ }^{(1)} T_{\alpha}+2 \sigma_{2}{ }^{(2)} T^{\alpha} \wedge{ }^{(3)} T_{\alpha}\right) .
\end{aligned}
$$

By noticing the trace and symmetry properties of the irreducible decompositions of curvature ${ }^{(3)} R_{\alpha \beta} \wedge \vartheta^{\alpha \beta}=R_{\alpha \beta} \wedge \vartheta^{\alpha \beta}$ and ${ }^{(6)} R^{\alpha \beta} \wedge \eta_{\alpha \beta}=R^{\alpha \beta} \wedge \eta_{\alpha \beta}$, more explicitly, we have

$$
\begin{aligned}
\left.V_{ \pm}\right|_{\text {weak }}= & \frac{1}{2 \kappa}\left(-a_{0} R^{\alpha \beta} \wedge \eta_{\alpha \beta}-2 \lambda_{0} \eta-b_{0} R_{\alpha \beta} \wedge \vartheta^{\alpha \beta}+a_{1}{ }^{(1)} T^{\alpha} \wedge^{\star(1)} T_{\alpha}\right. \\
& \left.+a_{2}{ }^{(2)} T^{\alpha} \wedge^{\star(2)} T_{\alpha}+a_{3}{ }^{(3)} T^{\alpha} \wedge^{\star(3)} T_{\alpha}+2 \sigma_{1}{ }^{(1)} T^{\alpha} \wedge{ }^{(1)} T_{\alpha}+2 \sigma_{2}{ }^{(2)} T^{\alpha} \wedge^{(3)} T_{\alpha}\right) .
\end{aligned}
$$

This lagrangian 4-form is equivalent to

$$
\begin{aligned}
\left.V_{ \pm}\right|_{\text {weak }}= & \frac{1}{2 \kappa}\left(-a_{0} R^{\alpha \beta} \wedge \eta_{\alpha \beta}-2 \lambda_{0} \eta-b_{0} R_{\alpha \beta} \wedge \vartheta^{\alpha \beta}+k_{1} T^{\alpha} \wedge^{\star} T_{\alpha}\right. \\
& \left.\left.+k_{2}\left(e_{\alpha}\right\lrcorner T^{\alpha}\right) \wedge^{\star}\left(e_{\beta}\right\lrcorner T^{\beta}\right)+k_{3}\left(\vartheta_{\alpha} \wedge T^{\alpha}\right) \wedge^{\star}\left(\vartheta_{\beta} \wedge T^{\beta}\right) \\
& \left.\left.+2 \ell_{1} T^{\alpha} \wedge T_{\alpha}+2 \ell_{2}\left(e_{\alpha}\right\lrcorner T^{\alpha}\right) \wedge\left(\vartheta_{\beta} \wedge T^{\beta}\right)\right) .
\end{aligned}
$$

under the redefinition of the coupling coefficients

$$
a_{1}=k_{1}, a_{2}=k_{1}+3 k_{2}, a_{3}=k_{1}+3 k_{3}, \sigma_{1}=\ell_{1}, \sigma_{2}=2 \ell_{1}-3 \ell_{2} .
$$

This correspondence was checked by Reduce-Excalc [13],[14]. The term with $b_{0}$ or $\sigma_{1}$ in (17) or, equivalently, that with $b_{0}$ or $\ell_{1}$ in (18) can be dropped by using the parity odd boundary term, the so-called Nieh-Yan 4-form [15],[16], $B_{T T}^{-}=d C_{T T}^{-}=\frac{1}{2} d\left(\vartheta^{\alpha} \wedge T_{\alpha}\right)=$ $\frac{1}{2}\left(T_{\alpha} \wedge T^{\alpha}+R_{\alpha \beta} \wedge \vartheta^{\alpha \beta}\right)$. We will choose $\sigma_{1}=\ell_{1}=0$ without loss of generality.

\section{Dirac Lagrangian}

We are using the formalism of Clifford algebra $\mathcal{C} \ell_{1,3}$-valued exterior forms. The $\mathcal{C} \ell_{1,3}$ algebra is generated by the relation among the orthonormal basis $\left\{\gamma_{0}, \gamma_{1}, \gamma_{2}, \gamma_{3}\right\}$

$$
\gamma^{\alpha} \gamma^{\beta}+\gamma^{\beta} \gamma^{\alpha}=2 g^{\alpha \beta}
$$


One particular representation of the $\gamma^{\alpha}$ 's is given by the following Dirac matrices

$$
\gamma_{0}=i\left(\begin{array}{cc}
-I & 0 \\
0 & I
\end{array}\right), \gamma_{1}=i\left(\begin{array}{cc}
0 & \sigma^{1} \\
-\sigma^{1} & 0
\end{array}\right), \gamma_{2}=i\left(\begin{array}{cc}
0 & \sigma^{2} \\
-\sigma^{2} & 0
\end{array}\right), \gamma_{3}=i\left(\begin{array}{cc}
0 & \sigma^{3} \\
-\sigma^{3} & 0
\end{array}\right) \text {, }
$$

where $\sigma^{1}, \sigma^{2}, \sigma^{3}$ are the Pauli matrices. In this case a Dirac spinor $\Psi$ can be represented by a 4-component column matrix. Thus we write explicitly the covariant exterior derivative of $\Psi$ and the quantity $D^{2} \Psi$

$$
D \Psi=d \Psi-\frac{1}{2} \Gamma^{\alpha \beta} \sigma_{\alpha \beta} \Psi, \quad D^{2} \Psi=-\frac{1}{2} R^{\alpha \beta} \sigma_{\alpha \beta} \Psi
$$

where $\sigma_{\alpha \beta}:=\frac{1}{4}\left[\gamma_{\alpha}, \gamma_{\beta}\right]$ are the generators of the Lorentz group [17],[18]. The Dirac adjoint is $\bar{\Psi}:=\Psi^{\dagger} \gamma_{0}$. Some relations of the Dirac matrices are

$$
\begin{aligned}
\sigma_{\alpha \beta} \gamma_{\gamma} & =\gamma_{\gamma} \sigma_{\alpha \beta}+g_{\beta \gamma} \gamma_{\alpha}-g_{\alpha \gamma} \gamma_{\beta}, \\
\sigma_{\alpha \beta} \gamma_{\gamma}+\gamma_{\gamma} \sigma_{\alpha \beta} & =-\epsilon_{\alpha \beta \gamma \delta} \gamma^{\delta} \gamma_{5}, \\
\gamma_{\gamma} \sigma_{\alpha \beta} & =\frac{1}{2} g_{\alpha \gamma} \gamma_{\beta}-\frac{1}{2} g_{\beta \gamma} \gamma_{\alpha}-\frac{1}{2} \epsilon_{\alpha \beta \gamma \delta} \gamma^{\delta} \gamma_{5} \\
{\left[\sigma_{\alpha \beta}, \sigma_{\gamma \delta}\right] } & =-g_{\alpha \gamma} \sigma_{\beta \delta}-g_{\beta \delta} \sigma_{\alpha \gamma}+g_{\alpha \delta} \sigma_{\beta \gamma}+g_{\beta \gamma} \sigma_{\alpha \delta},
\end{aligned}
$$

where $\gamma_{5}:=\gamma_{0} \gamma_{1} \gamma_{2} \gamma_{3}$. In order to obtain the Bjorken-Drell conventions [19] one has to replace $\gamma^{\alpha} \rightarrow-i \gamma^{\alpha}$ and $\gamma_{5} \rightarrow i \gamma_{5}$. Now we take the non-minimally coupled Dirac lagrangian given by the hermitian 4-form [20], [21]

$$
L_{\mathrm{D}}\left(g_{\alpha \beta}, \vartheta^{\alpha}, \Psi, D \Psi\right)=\frac{i \hbar}{2}\left[(1-i \alpha) \bar{\Psi}^{\star} \gamma \wedge D \Psi+(1+i \alpha) D \bar{\Psi} \wedge^{\star} \gamma \Psi\right]+i m c \bar{\Psi} \Psi \eta
$$

where $\gamma:=\gamma_{\alpha} \vartheta^{\alpha}$ and $\alpha$ is a real constant. The value $\alpha=0$ corresponds to the conventional minimal coupling of fermions to gravity. In general, an arbitrary real value for $\alpha$ corresponds to a non-minimal coupling. The coframe $\vartheta^{\alpha}$ necessarily occurs in the Dirac Lagrangian, even

in special relativity. The hermiticity of the lagrangian (24) leads to a charge current which admits the usual probabilistic interpretation.

\section{TOTAL LAGRANGIAN AND FIELD EQUATIONS}

In this work we will consider the total lagrangian

$$
L_{\text {tot }}=\left.V_{ \pm}\right|_{\text {weak }}\left(g_{\alpha \beta}, \vartheta^{\alpha}, T^{\alpha}, R^{\alpha \beta}\right)+L_{\mathrm{D}}\left(g_{\alpha \beta}, \vartheta^{\alpha}, \Psi, D \Psi\right)
$$


Then from (18) we compute the translation and the Lorentz excitations, respectively,

$$
\begin{aligned}
H_{\alpha} & \left.\left.:=-\frac{\partial V}{\partial T^{\alpha}}=-\frac{1}{\kappa}\left(k_{1}^{\star} T_{\alpha}+k_{2} e_{\alpha}\right\lrcorner^{\star} \mathcal{V}+k_{3} \vartheta_{\alpha} \wedge \mathcal{A}+\ell_{2} e_{\alpha}\right\lrcorner^{\star} \mathcal{A}-\ell_{2} \vartheta_{\alpha} \wedge \mathcal{V}\right), \\
H_{\alpha \beta} & :=-\frac{\partial V}{\partial R^{\alpha \beta}}=\frac{1}{2 \kappa}\left(a_{0} \eta_{\alpha \beta}+b_{0} \vartheta_{\alpha \beta}\right),
\end{aligned}
$$

where $\left.\mathcal{V}=e_{\alpha}\right\lrcorner T^{\alpha}$ and $\mathcal{A}={ }^{\star}\left(\vartheta_{\alpha} \wedge T^{\alpha}\right)$. Then, after calculating from (18), (26) and (27) the gauge currents of energy-momentum and spin

$$
\begin{aligned}
E_{\alpha} & \left.\left.\left.:=e_{\alpha}\right\lrcorner V+\left(e_{\alpha}\right\lrcorner T^{\beta}\right) \wedge H_{\beta}+\left(e_{\alpha}\right\lrcorner R^{\beta \gamma}\right) \wedge H_{\beta \gamma}, \\
E_{\alpha \beta} & :=-\frac{1}{2}\left(\vartheta_{\alpha} \wedge H_{\beta}-\vartheta_{\beta} \wedge H_{\alpha}\right),
\end{aligned}
$$

respectively, we can write the FIRST field equation $D H_{\alpha}-E_{\alpha}=\Sigma_{\alpha}$ as

$$
\begin{array}{r}
\frac{a_{0}}{2} R^{\beta \gamma} \wedge \eta_{\alpha \beta \gamma}+\lambda_{0} \eta_{\alpha}+b_{0} R_{\alpha \beta} \wedge \vartheta^{\beta} \\
\left.\left.-k_{1} D^{\star} T_{\alpha}-\frac{k_{1}}{2}\left[e_{\alpha}\right\lrcorner\left(T_{\beta} \wedge{ }^{\star} T^{\beta}\right)-2\left(e_{\alpha}\right\lrcorner T^{\beta}\right) \wedge{ }^{\star} T_{\beta}\right] \\
\left.\left.\left.\left.-k_{2} D\left(e_{\alpha}\right\lrcorner^{\star} \mathcal{V}\right)-\frac{k_{2}}{2}\left[e_{\alpha}\right\lrcorner\left(\mathcal{V} \wedge{ }^{\star} \mathcal{V}\right)-2\left(e_{\alpha}\right\lrcorner T^{\beta}\right) \wedge\left(e_{\beta}\right\lrcorner^{\star} \mathcal{V}\right)\right] \\
\left.\left.-k_{3} D\left(\vartheta_{\alpha} \wedge \mathcal{A}\right)+\frac{k_{3}}{2}\left[e_{\alpha}\right\lrcorner\left(\mathcal{A} \wedge{ }^{\star} \mathcal{A}\right)+2\left(e_{\alpha}\right\lrcorner T^{\beta}\right) \wedge\left(\vartheta_{\beta} \wedge \mathcal{A}\right)\right] \\
\left.\left.\left.\left.-\ell_{2} D\left(e_{\alpha}\right\lrcorner^{\star} \mathcal{A}-\vartheta_{\alpha} \wedge \mathcal{V}\right)-\ell_{2}\left[e_{\alpha}\right\lrcorner\left(\mathcal{V} \wedge{ }^{\star} \mathcal{A}\right)-\left(e_{\alpha}\right\lrcorner T^{\beta}\right) \wedge\left(e_{\beta}\right\lrcorner^{\star} \mathcal{A}-\vartheta_{\beta} \wedge \mathcal{V}\right)\right]=\kappa \Sigma_{\alpha},(30
\end{array}
$$

and the SECOND field equation $D H_{\alpha \beta}-E_{\alpha \beta}=\tau_{\alpha \beta}$ as

$$
\begin{aligned}
\frac{a_{0}}{2} T^{\gamma} \wedge \eta_{\alpha \beta \gamma}-\frac{b_{0}}{2}\left(\vartheta_{\alpha} \wedge T_{\beta}-\vartheta_{\beta} \wedge T_{\alpha}\right)-\frac{k_{1}}{2}\left(\vartheta_{\alpha} \wedge{ }^{\star} T_{\beta}-\vartheta_{\beta} \wedge{ }^{\star} T_{\alpha}\right) \\
\left.\left.-\frac{k_{2}}{2}\left[\vartheta_{\alpha} \wedge\left(e_{\beta}\right\lrcorner^{\star} \mathcal{V}\right)-\vartheta_{\beta} \wedge\left(e_{\alpha}\right\lrcorner^{\star} \mathcal{V}\right)\right]-k_{3} \vartheta_{\alpha \beta} \wedge \mathcal{A} \\
\left.\left.-\frac{\ell_{2}}{2}\left[\vartheta_{\alpha} \wedge\left(e_{\beta}\right\lrcorner^{\star} \mathcal{A}\right)-\vartheta_{\beta} \wedge\left(e_{\alpha}\right\lrcorner^{\star} \mathcal{A}\right)-2 \vartheta_{\alpha \beta} \wedge \mathcal{V}\right]=\kappa \tau_{\alpha \beta},
\end{aligned}
$$

where the Dirac energy-momentum current and the Dirac spin current are, respectively,

$$
\begin{aligned}
\Sigma_{\alpha} & =\frac{i \hbar}{2}\left[(1-i \alpha) \bar{\Psi}^{\star}\left(\gamma \wedge \vartheta_{\alpha}\right) \wedge D \Psi-(1+i \alpha) D \bar{\Psi} \wedge^{\star}\left(\gamma \wedge \vartheta_{\alpha}\right) \Psi\right]+i m c \bar{\Psi} \Psi \eta_{\alpha} \\
\tau_{\alpha \beta} & =\frac{i \hbar}{4}\left[\vartheta_{\alpha \beta} \wedge \bar{\Psi} \gamma \gamma_{5} \Psi+i \alpha \bar{\Psi}\left(\gamma_{\alpha} \eta_{\beta}-\gamma_{\beta} \eta_{\alpha}\right) \Psi\right]
\end{aligned}
$$

Here it would be useful to remark that the FIRST field equation is obtained by varying the total lagrangian 4-form (25) with respect to $\vartheta^{\alpha}$ and the SECOND field equation with respect to $\Gamma^{\alpha \beta}$ [6],[9]. Finally, the variation of (25) with respect to $\bar{\Psi}$ yields the Dirac equation

$$
{ }^{\star} \gamma \wedge\left(D-\frac{1+i \alpha}{2} \mathcal{V}\right) \Psi+\frac{m c}{\hbar} \Psi \eta=0
$$


This equation can be decomposed into a Riemannian part plus a torsional part by simple algebra. Firstly, by noting $\mathcal{V}=K^{\alpha \beta}{ }_{\alpha} \vartheta_{\beta}$, we rewrite it as

$$
{ }^{\star} \gamma \wedge \widetilde{D} \Psi+\frac{m c}{\hbar} \Psi \eta-\frac{1}{2}\left(K^{\alpha \beta \mu} \gamma_{\mu} \sigma_{\alpha \beta}-(1+i \alpha) K_{\alpha \beta}^{\alpha \beta} \gamma_{\beta}\right) \Psi \eta=0,
$$

where $K_{\alpha \beta}=K_{\alpha \beta \gamma} \vartheta^{\gamma}$. After using the third relation of (23), we obtain

$$
{ }^{\star} \gamma \wedge \widetilde{D} \Psi+\left(\frac{m c}{\hbar}-\frac{1}{4} \mathcal{A}_{\alpha} \gamma^{\alpha} \gamma_{5}+\frac{i \alpha}{2} \mathcal{V}_{\alpha} \gamma^{\alpha}\right) \Psi \eta=0
$$

with $\left.e_{\alpha}\right\lrcorner \mathcal{V}:=\mathcal{V}_{\alpha}=K_{\beta \alpha}{ }^{\beta}$ and $\left.e_{\alpha}\right\lrcorner \mathcal{A}:=\mathcal{A}_{\alpha}=\epsilon_{\alpha \beta \mu \nu} K^{\beta \mu \nu}$. This means that in the minimally coupled Dirac equation only the totally antisymmetric axial component of the torsion survives. Now, we want to reexpress (32) in a different way by means of (34) and its adjoint, $\left(D-\frac{1-i \alpha}{2} \mathcal{V}\right) \bar{\Psi} \wedge{ }^{\star} \gamma+\frac{m c}{\hbar} \bar{\Psi} \eta=0$,
$\Sigma_{\alpha}=\frac{i \hbar}{2}\left[(1-i \alpha) \bar{\Psi} \gamma_{\beta}\left(D_{\alpha} \Psi\right)-(1+i \alpha)\left(D_{\alpha} \bar{\Psi}\right) \gamma_{\beta} \Psi\right] \eta^{\beta}$

where $\left.D_{\alpha}:=e_{\alpha}\right\lrcorner D$. Thus we can display the canonical energy-momentum tensor, $\Sigma_{\alpha}:=$ $\mathcal{T}_{\alpha \beta} \eta^{\beta}$, for a Dirac field as $\mathcal{T}_{\alpha \beta}=\frac{i \hbar}{2}\left[(1-i \alpha) \bar{\Psi} \gamma_{\beta}\left(D_{\alpha} \Psi\right)-(1+i \alpha)\left(D_{\alpha} \bar{\Psi}\right) \gamma_{\beta} \Psi\right]$. This version of the Dirac energy-momentum may be more familiar from the literature [22], [23], [24].

The next step is to deal with the SECOND equation (31). But, before that, we will review the symmetry properties of the spin current 3-form. Only the first part of the Dirac spin current 3 -form vanishes, i.e. ${ }^{(1)} \tau_{\alpha \beta}=0$, but ${ }^{(2)} \tau_{\alpha \beta} \neq 0$ and ${ }^{(3)} \tau_{\alpha \beta} \neq 0$, see the appendix for details. Then, by denoting the Dirac spin current pseudovector and vector, respectively, as

$$
S^{\alpha}:=i \bar{\Psi} \gamma^{\alpha} \gamma_{5} \Psi, \quad W^{\alpha}:=i \bar{\Psi} \gamma^{\alpha} \Psi
$$

we can calculate $T^{\alpha}$ from the SECOND equation (31)

$$
\left.T^{\alpha}=2 \kappa\left[\vartheta^{\alpha} \wedge\left(c_{2} S+d_{2} W\right)+e^{\alpha}\right\lrcorner^{\star}\left(c_{3} S+d_{3} W\right)\right]
$$

where $S=S^{\alpha} \vartheta_{\alpha}, W=W^{\alpha} \vartheta_{\alpha}$,

$$
\begin{aligned}
& c_{2}=-\hbar\left(b_{0}+3 \ell_{2}\right) / \mathcal{D}, \quad d_{2}=i \hbar \alpha\left(a_{0}-2 k_{1}-6 k_{3}\right) / \mathcal{D}, \\
& c_{3}=\hbar\left(2 a_{0}-k_{1}+3 k_{2}\right) / \mathcal{D}, \quad d_{3}=2 i \hbar \alpha\left(b_{0}-3 \ell_{2}\right) / \mathcal{D}
\end{aligned}
$$

with $^{1}$

$$
\mathcal{D}=4\left[a_{0}\left(2 a_{0}-5 k_{1}+3 k_{2}-12 k_{3}\right)+2 k_{1}\left(k_{1}-3 k_{2}+3 k_{3}\right)-18 k_{2} k_{3}+2 b_{0}^{2}-18 \ell_{2}^{2}\right]
$$

\footnotetext{
${ }^{1}$ Incidentally, the constants $d_{2}$ and $d_{3}$ should not be confused with $\left.e_{2}\right\lrcorner d$ and $\left.e_{3}\right\lrcorner d$, repectively.
} 
This result has been checked by the computer algebra system Reduce and its package Excalc [13],[14]. For the Bjorken-Drell conventions $S^{\alpha} \rightarrow-S^{\alpha}$ and $W^{\alpha} \rightarrow-i W^{\alpha}$. Now, by calculating $\mathcal{V}=6 \kappa\left(c_{2} S+d_{2} W\right)$ and $\mathcal{A}=6 \kappa\left(c_{3} S+d_{3} W\right)$, we obtain the irreducible parts of the torsion as

$$
\left.{ }^{(1)} T^{\alpha}=0,{ }^{(2)} T^{\alpha}=2 \kappa \vartheta^{\alpha} \wedge\left(c_{2} S+d_{2} W\right),{ }^{(3)} T^{\alpha}=2 \kappa e^{\alpha}\right\lrcorner^{\star}\left(c_{3} S+d_{3} W\right) .
$$

Therefore we can drop $a_{1}$ (or $k_{1}$ ) without loss of generality. So, we will continue with non-vanishing coupling constants $\alpha, a_{0}, \lambda_{0}, b_{0}, k_{2}=a_{2} / 3, k_{3}=a_{3} / 3, \ell_{2}=-\sigma_{2} / 3$. Thus,

$$
\begin{aligned}
c_{2} & =-\hbar\left(b_{0}+3 \ell_{2}\right) / \mathfrak{D} & & (\text { pseudoscalar }), \\
d_{2} & =i \hbar \alpha\left(a_{0}-6 k_{3}\right) / \mathfrak{D} & & (\text { scalar }), \\
c_{3} & =\hbar\left(2 a_{0}+3 k_{2}\right) / \mathfrak{D} & & (\text { scalar }), \\
d_{3} & =2 i \hbar \alpha\left(b_{0}-3 \ell_{2}\right) / \mathfrak{D} & & \text { (pseudoscalar) },
\end{aligned}
$$

with

$$
\mathfrak{D}=4\left[a_{0}\left(2 a_{0}+3 k_{2}-12 k_{3}\right)-18 k_{2} k_{3}+2 b_{0}^{2}-18 \ell_{2}^{2}\right] .
$$

Here we recall that the coefficients $a_{0}, \lambda_{0}, k_{1}, k_{2}, k_{3}$ are scalars (since they contain an odd number of Hodge stars) and the remaining ones $b_{0}, \ell_{1}, \ell_{2}$ are pseudoscalars (containing an even number of Hodge stars). Because the denominator contains squares of $b_{0}$ and $\ell_{2}$, it is a scalar quantity. Thus, as $c_{2}$ and $d_{3}$ are pseudoscalars, $d_{2}$ and $c_{3}$ are scalars. If we put the pseudoscalar coefficients to zero, $b_{0}=\ell_{2}=0$, then we are left only with the scalars $d_{2}$ and $c_{3}$ as a consistent result. We also remark that for a viable theory the constants have to be constrained by studying the propagating modes (see Chen et al [25]). It is clear that a vanishing $\mathfrak{D}$ would disqualify the model from being physical. In other words, a closer investigation has to get information also on the behavior of $\mathfrak{D}$.

The next step is to rewrite the Dirac equation (35) in terms of Riemannian quantities. The first job is to calculate the contortion via (11)

$$
K_{\alpha \beta}=\kappa\left[2 c_{2}\left(S_{\beta} \vartheta_{\alpha}-S_{\alpha} \vartheta_{\beta}\right)+2 d_{2}\left(W_{\beta} \vartheta_{\alpha}-W_{\alpha} \vartheta_{\beta}\right)-c_{3}{ }^{\star}\left(\vartheta_{\alpha \beta} \wedge S\right)-d_{3}{ }^{\star}\left(\vartheta_{\alpha \beta} \wedge W\right)\right]
$$

Then the Dirac equation (35) turns out to be

$$
{ }^{\star} \gamma \wedge \widetilde{D} \Psi+\left\{\frac{m c}{\hbar}-\frac{3 \kappa}{4}\left[S_{\alpha} \gamma^{\alpha}\left(c_{3} \gamma_{5}-4 i \alpha c_{2}\right)+W_{\alpha} \gamma^{\alpha}\left(d_{3} \gamma_{5}-4 i \alpha d_{2}\right)\right]\right\} \Psi \eta=0 .
$$


We obtained the parity-conserving terms $\sim c_{3} S_{\alpha} \gamma^{\alpha} \gamma_{5} \Psi$ and $\sim 4 i \alpha d_{2} W_{\alpha} \gamma^{\alpha} \Psi$, and the parityviolating term $\sim \gamma^{\alpha}\left(-4 i \alpha c_{2} S_{\alpha}+d_{3} \gamma_{5} W_{\alpha}\right) \Psi$. But for $\alpha=0$, the parity-violating term and the second one of the parity conserving terms drop out and we are left with only the first parity-conserving term.

Finally we split the FIRST equation into a Riemann part and a torsion part. Let us first decompose the curvature 2-form as Riemann 2-form plus contortion terms

$$
\begin{aligned}
R_{\alpha}{ }^{\beta} & =d\left(\widetilde{\Gamma}_{\alpha}^{\beta}-K_{\alpha}^{\beta}\right)-\left(\widetilde{\Gamma}_{\alpha}^{\gamma}-K_{\alpha}^{\gamma}\right) \wedge\left(\widetilde{\Gamma}_{\gamma}^{\beta}-K_{\gamma}{ }^{\beta}\right) \\
& =\widetilde{R}_{\alpha}{ }^{\beta}-\widetilde{D} K_{\alpha}{ }^{\beta}-K_{\alpha}^{\gamma} \wedge K_{\gamma}{ }^{\beta}
\end{aligned}
$$

Then, with the Einstein 3-form $\widetilde{G}_{\alpha}=\frac{1}{2} \widetilde{R}^{\beta \gamma} \wedge \eta_{\alpha \beta \gamma}$, we can write the FIRST equation (30) in terms of Riemannian quantities plus Dirac field

$$
\begin{aligned}
a_{0} \widetilde{G}_{\alpha}+\lambda_{0} \eta_{\alpha}= & \kappa\left[\widetilde{\Sigma}_{\alpha}-\frac{\hbar}{4}\left(\widetilde{D} S^{\beta}\right) \wedge \vartheta_{\alpha \beta}-\frac{i \alpha \hbar}{2}\left(\widetilde{D} W^{\beta}\right) \wedge \eta_{\alpha \beta}\right] \\
& +\kappa^{2}\left[C_{S} S_{\beta} S^{\beta}+C_{W} W_{\beta} W^{\beta}+C_{S W} S_{\beta} W^{\beta}\right] \eta_{\alpha}
\end{aligned}
$$

where

$$
\begin{gathered}
C_{S}=-a_{0}\left(4 c_{2}^{2}-c_{3}^{2}\right)+30 k_{2} c_{2}^{2}-6 k_{3} c_{3}^{2}-\hbar c_{3}-4 c_{2} c_{3}\left(b_{0}-9 \ell_{2}\right), \\
C_{W}=-a_{0}\left(4 d_{2}^{2}-d_{3}^{2}\right)+30 k_{2} d_{2}^{2}-6 k_{3} d_{3}^{2}+2 i \alpha \hbar d_{2}-4 d_{2} d_{3}\left(b_{0}+9 \ell_{2}\right), \\
C_{S W}=-2 a_{0}\left(4 c_{2} d_{2}-c_{3} d_{3}\right)+60 k_{2} c_{2} d_{2}-12 k_{3} c_{3} d_{3}-\hbar d_{3}+2 i \alpha \hbar c_{2} \\
-4\left(d_{2} c_{3}+c_{2} d_{3}\right)\left(b_{0}-9 \ell_{2}\right) .
\end{gathered}
$$

Whereas $C_{S}, C_{W}$ are scalars, $C_{S W}$ is a pseudo scalar. In (48) we decomposed the Dirac energy-momentum current (32) according to

$$
\begin{aligned}
\Sigma_{\alpha} & =\widetilde{\Sigma}_{\alpha}-\frac{\kappa \hbar}{2}\left\{c_{3}\left(2 S_{\beta} S^{\beta} \eta_{\alpha}+S_{\alpha} S_{\beta} \eta^{\beta}\right)+d_{3}\left(2 S_{\beta} W^{\beta} \eta_{\alpha}+S_{\alpha} W_{\beta} \eta^{\beta}\right)+2 d_{2} S^{\beta} W^{\gamma} \vartheta_{\alpha \beta \gamma}\right. \\
& \left.-i \alpha\left[2 d_{2}\left(2 W_{\beta} W^{\beta} \eta_{\alpha}+W_{\alpha} W_{\beta} \eta^{\beta}\right)+2 c_{2}\left(2 S_{\beta} W^{\beta} \eta_{\alpha}+W_{\alpha} S_{\beta} \eta^{\beta}\right)+c_{3} S^{\beta} W^{\gamma} \vartheta_{\alpha \beta \gamma}\right]\right\},
\end{aligned}
$$

with

$$
\widetilde{\Sigma}_{\alpha}=\frac{i \hbar}{2}\left[(1-i \alpha) \bar{\Psi} \gamma_{\beta}\left(\widetilde{D}_{\alpha} \Psi\right)-(1+i \alpha)\left(\widetilde{D}_{\alpha} \bar{\Psi}\right) \gamma_{\beta} \Psi\right] \eta^{\beta}
$$

Now we note that the Riemannian field equations (46) and (48) are derived from the (hermitian) lagrangian 4-form $L_{\text {tot }}=V_{E H \lambda}+L_{D}+L_{\text {int }}+\Lambda_{\alpha} \wedge T^{\alpha}$ where the lagrange 
multiplier $\Lambda_{\alpha}$ is a 2-form, $L_{D}$ is the non-minimally coupled the Dirac lagrangian given by (24), $V_{E H \lambda}$ is the Einstein-Hilbert lagrangian with the cosmological constant

$$
V_{E H \lambda}=-\frac{a_{0}}{2 \kappa} R^{\alpha \beta} \wedge \eta_{\alpha \beta}-\frac{\lambda_{0}}{\kappa} \eta
$$

and $L_{\mathrm{int}}$ is the interaction lagrangian

$$
L_{\text {int }}=\kappa\left(E_{S} S_{\alpha} S^{\alpha}+E_{W} W_{\alpha} W^{\alpha}+E_{S W} S_{\alpha} W^{\alpha}\right) \eta,
$$

with the coefficients

$$
\begin{gathered}
E_{S}=-3 a_{0}\left(4 c_{2}^{2}-c_{3}^{2}\right)-12 b_{0} c_{2} c_{3}+18 k_{2} c_{2}^{2}-18 k_{3} c_{3}^{2}+36 \ell_{2} c_{2} c_{3}-3 \hbar c_{3} / 2, \\
E_{W}=-3 a_{0}\left(4 d_{2}^{2}-d_{3}^{2}\right)-12 b_{0} d_{2} d_{3}+18 k_{2} d_{2}^{2}-18 k_{3} d_{3}^{2}+36 \ell_{2} d_{2} d_{3}+3 i \alpha \hbar d_{2}, \\
E_{S W}=-6 a_{0}\left(4 c_{2} d_{2}-c_{3} d_{3}\right)-12 b_{0}\left(c_{2} d_{3}+d_{2} c_{3}\right)+36 k_{2} c_{2} d_{2}-36 k_{3} c_{3} d_{3} \\
\quad+36 \ell_{2}\left(c_{2} d_{3}+d_{2} c_{3}\right)-3 \hbar d_{3} / 2+3 i \alpha \hbar c_{2} .
\end{gathered}
$$

\section{The Einstein-Dirac Theory with $\lambda_{0}$}

For comparison with the literature, we want to summarize briefly the minimally coupled Einstein-Dirac theory with cosmological constant. It is defined by the total lagrangian 4form $L_{\text {tot }}=V_{E H \lambda}+L_{D}+\Lambda_{\alpha} \wedge T^{\alpha}$ where $V_{E H \lambda}$ is given by (54), $L_{D}$ is the minimally coupled Dirac lagrangian that is given by (24) with $\alpha=0$ and $\Lambda_{\alpha}$ is the lagrange multiplier 2-form whose variation yields $T^{\alpha}=0$. The excitations are calculated as

$$
H_{\alpha}=0, \quad H_{\alpha \beta}=\frac{a_{0}}{2 \kappa} \eta_{\alpha \beta} .
$$

Then the gauge currents are obtained as

$$
E_{\alpha}=-\frac{a_{0}}{2 \kappa} R^{\beta \gamma} \wedge \eta_{\alpha \beta \gamma}-\frac{\lambda_{0}}{\kappa} \eta_{\alpha}, \quad E_{\alpha \beta}=0 .
$$

Since the torsion is zero, all the concerned Riemannian quantities will be marked by a tilde. Now we write down the modified FIRST equation, $D H_{\alpha}-E_{\alpha}-D \Lambda_{\alpha}=\Sigma_{\alpha}$, the modified SECOND equation, $D H_{\alpha \beta}-E_{\alpha \beta}-\frac{1}{2}\left(\vartheta_{\alpha} \wedge \Lambda_{\beta}-\vartheta_{\beta} \wedge \Lambda_{\alpha}\right)=\tau_{\alpha \beta}$ and the Dirac equation, respectively

$$
\begin{aligned}
\frac{a_{0}}{2 \kappa} \widetilde{R}^{\beta \gamma} \wedge \eta_{\alpha \beta \gamma}+\frac{\lambda_{0}}{\kappa} \eta_{\alpha}-\widetilde{D} \Lambda_{\alpha} & =\widetilde{\Sigma}_{\alpha}, \\
\frac{a_{0}}{2 \kappa} \widetilde{D} \eta_{\alpha \beta}-\frac{1}{2}\left(\vartheta_{\alpha} \wedge \Lambda_{\beta}-\vartheta_{\beta} \wedge \Lambda_{\alpha}\right) & =\frac{\hbar}{4} S^{\gamma} \vartheta_{\alpha \beta \gamma}, \\
{ }^{\star} \gamma \wedge \widetilde{D} \Psi+\frac{m c}{\hbar} \Psi \eta & =0
\end{aligned}
$$


where the Riemannian Dirac energy-momentum current $\widetilde{\Sigma}_{\alpha}$ is the same as (53) with $\alpha=0$. By using the identity $\widetilde{D} \eta_{\alpha \beta}=0$ we calculate $\Lambda_{\alpha}=-\frac{\hbar}{4} S^{\beta} \vartheta_{\alpha \beta}$ from (62). The substitution of this into (61) gives rise to the following

$$
a_{0} \widetilde{G}_{\alpha}+\lambda_{0} \eta_{\alpha}=\kappa \widetilde{\Sigma}_{\alpha}-\frac{\kappa \hbar}{4}\left(\widetilde{D} S^{\beta}\right) \wedge \vartheta_{\alpha \beta}
$$

\section{CONCLUDING REMARKS}

In this work we considered the Dirac field non-minimally coupled to the gravitational field in the framework of the Poincare gauge theory. The gravitational lagrangian contains terms at most linear in curvature and quadratic in torsion, both of odd and even parity. This is the most general $R+T^{2}$-type lagrangian with eight parameters, $\alpha, \lambda_{0}, a_{0}, b_{0}, k_{1}, k_{2}, k_{3}, \ell_{2}$, all together. The field equation, obtained by varying the total lagrangian with respect to the Lorentz connection, was solved with respect to torsion in terms of the Dirac vector and the Dirac axial vector (38). Our first result is that as the tensor piece of the torsion, ${ }^{(1)} T^{\alpha}$, is zero and the vector part, ${ }^{(2)} T^{\alpha}$, and the axial part, ${ }^{(3)} T^{\alpha}$, are nonzero. Thus we set $k_{1}=0$ without loss of generality and the number of the parameters of our model decreased to seven. However, this is still the most general $R+T^{2}$ model in the literature. Then by using the decomposition of the Lorentz connection in terms of its Riemannian piece and contortion, the Dirac equation is decomposed into a Riemannian piece and torsional terms, see eqn(46). Here we observe that the parity-violating term $\sim \gamma^{\alpha}\left(S_{\alpha} \pm \gamma_{5} W_{\alpha}\right) \Psi$ is proportional to non-minimal coupling parameter, $\alpha$. After that we applied the same strategy to the field equation obtained by variation with respect to the orthonormal coframe, see eqn(48). Consequently, the FIRST equation turned out to be the form of the familiar Einstein-Dirac equation plus correction terms. Then we obtained a Riemannian lagrangian 4-form equivalent to our Poincare gauge theory of gravity. Finally we summarized the minimally coupled Einstein-Dirac theory with $\lambda_{0}$ for easy comparison of our results with the literature. Accordingly when $\alpha=0$ we realized that the relevant quantities are $c_{3}$ given by (42) and $C_{S}$ given by (49). Interestingly $c_{2}$ given by (40) does not appear explicitly in the Dirac equation (46) in this case. Thus the case of that $\alpha=0, c_{3}=0$ and $C_{S}=0$, including the possibility $k_{2}=-2 a_{0} / 3$ and $\ell_{2}=-b_{0} / 3$ as well, corresponds to the minimally coupled Einstein-Dirac theory. For the minimally coupled Einstein-Cartan-Dirac theory with cosmological constant $\left(\alpha=0, k_{2}=k_{3}=b_{0}=\ell_{2}=0\right)$ the relevant coefficients turn out to be $C_{S}=-3 \hbar^{2} / 16 a_{0}$ and $c_{3}=\hbar / 4 a_{0}$. In the minimally 
coupled weak Poincare gauge theory with even parity $\left(\alpha=b_{0}=\ell_{2}=0\right)$ they are nonzero. In the opposite case with odd parity $\left(\alpha=a_{0}=k_{2}=k_{3}=0\right)$ both $c_{3}$ and $C_{S}$ vanish. For our case they shift compared with the literature.

In Ref. [26] the authors reanalyzed the Hughes-Drever type experiments carried out for testing the anisotropy of mass and anomalous spin couplings and deduced a constraint on the axial torsion by $\left|S_{\alpha}\right| \leq 10^{-15} \mathrm{~m}^{-1}$. Since they considered minimal Dirac couplings $(\alpha=0)$ it was not possible to test other parts of the torsion tensor. Then they argue that one needs higher spin equations for a coupling to the trace and the traceless part of torsion tensor. Thus to repeat a similar analysis for our Dirac equation (46) may give insights on the constraints of vector components of torsion. A decade later Kostelecky et al exploited newer experimental searches (Zeeman measurements with a dual maser and studies of a spin-polarized torsion pendulum) for Lorentz violation in order to extract new constraints

on torsion components down to levels of order $10^{-15} \mathrm{~m}^{-1}$ [27]. Their analysis were performed through torsion nonminimal couplings to standard-model fields. Some combinations of our parameters correspond to their parameters $\xi_{1}^{(4)}, \xi_{2}^{(4)}, \xi_{3}^{(4)}, \xi_{4}^{(4)}$. In another work the authors discussed the possibility to perform and use the exact Foldy-Wouthuysen transformation for the Dirac spinor coupled to different CPT and Lorentz violating terms [28]. In accord with their result there may be a mixing between the magnetic and torsion fields, and the strongness of magnetic field may compensate the weakness of torsion. Consequently, the mixture term may affect the motion of a test particle in a notable way. When our work is compared with that of [28], it is realized that their four parameters $a_{\mu}, b_{\mu}, c^{\mu \nu}, d^{\mu \nu}$ are some combinations of ours.

\section{Acknowledgments}

The author thanks F.W. Hehl for reading the paper and for stimulating comments.

\section{Appendix A: Irreducible Decomposition of Torsion}

Torsion is decomposed as

$$
T^{\alpha}={ }^{(1)} T^{\alpha}+{ }^{(2)} T^{\alpha}+{ }^{(3)} T^{\alpha}
$$


in terms of

$$
\begin{aligned}
{ }^{(2)} T^{\alpha} & =-\frac{1}{3} \mathcal{V} \wedge \vartheta^{\alpha}, \\
{ }^{(3)} T^{\alpha} & =\frac{1}{3}{ }^{\star}\left(\mathcal{A} \wedge \vartheta^{\alpha}\right), \\
{ }^{(1)} T^{\alpha} & =T^{\alpha}-{ }^{(2)} T^{\alpha}-{ }^{(3)} T^{\alpha},
\end{aligned}
$$

where $\left.\mathcal{V}=e_{\alpha}\right\lrcorner T^{\alpha}$ and $\mathcal{A}={ }^{\star}\left(\vartheta_{\alpha} \wedge T^{\alpha}\right)$. The irreducible components of torsion satisfy

$$
\left.\left.{ }^{(1)} T^{\alpha} \wedge \vartheta_{\alpha}=0,{ }^{(2)} T^{\alpha} \wedge \vartheta_{\alpha}=0, \quad e_{\alpha}\right\lrcorner^{(1)} T^{\alpha}=0, \quad e_{\alpha}\right\lrcorner^{(3)} T^{\alpha}=0 .
$$

Thus the ${ }^{(I)} T^{\alpha}$ 's are orthogonal in the following two senses:

$$
\begin{aligned}
& { }^{(I)} T^{\alpha} \wedge{ }^{\star(J)} T_{\alpha}={ }^{(J)} T^{\alpha} \wedge{ }^{\star(I)} T_{\alpha}\left\{\begin{array}{l}
\neq 0 \text { if } I=J \\
=0 \text { if } I \neq J
\end{array}\right. \\
& { }^{(I)} T^{\alpha} \wedge{ }^{(J)} T_{\alpha}={ }^{(J)} T^{\alpha} \wedge{ }^{(I)} T_{\alpha}\left\{\begin{array}{l}
\neq 0 \text { if } I=J=1 \\
\neq 0 \text { if } I=2, J=3 \\
=0 \text { otherwise }
\end{array}\right.
\end{aligned}
$$

Appendix B: Irreducible Decomposition of Energy-momentum 3-form in 4 Dimensions

Any vector-valued 3 -form $\Sigma_{\alpha}$ in 4 dimensions can be decomposed as

$$
\Sigma_{\alpha}={ }^{(1)} \Sigma_{\alpha}+{ }^{(2)} \Sigma_{\alpha}+{ }^{(3)} \Sigma_{\alpha}
$$

in terms of

$$
\begin{aligned}
& { }^{(2)} \Sigma_{\alpha}=\frac{1}{2} \mathcal{F} \wedge \vartheta_{\alpha}, \\
& \left.{ }^{(3)} \Sigma_{\alpha}=-\frac{1}{4} e_{\alpha}\right\lrcorner \mathcal{G}, \\
& { }^{(1)} \Sigma_{\alpha}=\Sigma_{\alpha}-{ }^{(2)} \Sigma_{\alpha}-{ }^{(3)} \Sigma_{\alpha},
\end{aligned}
$$

where $\left.\mathcal{F}=e^{\alpha}\right\lrcorner \Sigma_{\alpha}$ and $\mathcal{G}=\Sigma_{\alpha} \wedge \vartheta^{\alpha}$. The irreducible parts satisfy

$$
\left.\left.{ }^{(1)} \Sigma^{\alpha} \wedge \vartheta_{\alpha}=0,{ }^{(2)} \Sigma^{\alpha} \wedge \vartheta_{\alpha}=0, \quad e_{\alpha}\right\lrcorner^{(1)} \Sigma^{\alpha}=0, \quad e_{\alpha}\right\lrcorner^{(3)} \Sigma^{\alpha}=0 .
$$




\section{Appendix C: Irreducible Decomposition of Spin 3-form in 4 Dimensions}

Any bivector-valued 3 -form $\tau_{\alpha \beta}=-\tau_{\beta \alpha}$ in 4 dimensions can be decomposed as

$$
\tau_{\alpha \beta}={ }^{(1)} \tau_{\alpha \beta}+{ }^{(2)} \tau_{\alpha \beta}+{ }^{(3)} \tau_{\alpha \beta}
$$

in terms of

$$
\begin{aligned}
& \left.\left.{ }^{(2)} \tau_{\alpha \beta}=\frac{1}{3}\left(e_{\alpha}\right\lrcorner \mathcal{W}_{\beta}-e_{\beta}\right\lrcorner \mathcal{W}_{\alpha}\right), \\
& { }^{(3)} \tau_{\alpha \beta}=-\frac{1}{6} \mathcal{B} \wedge \vartheta_{\alpha \beta}, \\
& { }^{(1)} \tau_{\alpha \beta}=\tau_{\alpha \beta}-{ }^{(2)} \tau_{\alpha \beta}-{ }^{(3)} \tau_{\alpha \beta},
\end{aligned}
$$

where $\mathcal{W}_{\alpha}=\tau_{\alpha \beta} \wedge \vartheta^{\beta}$ and $\left.\left.\mathcal{B}=e_{\alpha}\right\lrcorner e_{\beta}\right\lrcorner \tau^{\alpha \beta}$. The irreducible parts satisfy

$$
\left.\left.\left.\left.e_{\alpha}\right\lrcorner e_{\beta}\right\lrcorner^{(1)} \tau^{\alpha \beta}=0, \quad e_{\alpha}\right\lrcorner e_{\beta}\right\lrcorner^{(2)} \tau^{\alpha \beta}=0,{ }^{(1)} \tau^{\alpha \beta} \wedge \vartheta_{\beta}=0,{ }^{(3)} \tau^{\alpha \beta} \wedge \vartheta_{\beta}=0 .
$$

For more information on the decompositions of the related quantities one can consult [6].

[1] L. Freidel, D. Minic and T. Takeuchi, Quantum gravity, torsion, parity violation, and all that, Phys. Rev. D 72, 104002 (2005) [arXiv:hep-th/0507253].

[2] I. B. Khriplovich and A. A. Pomeransky, Remark on Immirzi Parameter, Torsion, and Discrete Symmetries, Phys. Rev. D 73107502 (2006) [arXiv:hep-th/0508136].

[3] K. Hayashi and T. Shirafuji, Gravity from Poincare Gauge Theory of the Fundamental Particles.I, Prog. Theor. Phys. 64866 (1980).

[4] M. Blagojevic and I. A. Nikolic, Hamiltonian dynamics of Poincare gauge theory: General structure in the time gauge, Phys. Rev. D 28, 2455 (1983).

[5] I. A. Nikolic, Dirac Hamiltonian structure of $R+R^{2}+T^{2}$ Poincare gauge theory of gravity without gauge fixing, Phys. Rev. D 30, 2508 (1984).

[6] P. Baekler, F. W. Hehl and J. M. Nester, Poincare gauge theory of gravity: Friedman cosmology with even and odd parity modes. Analytic part, Phys. Rev. D 83, 024001 (2011) [arXiv:1009.5112].

[7] I. L. Shapiro, Physical aspects of the space-time torsion, Phys. Rept. 357, 113 (2002) [arXiv:hep-th/0103093]. 
[8] A. J. Purcell, Pseudoscalar action in a Cartan spacetime, Phys. Rev. D 18, 2730 (1978).

[9] F. W. Hehl, J. D. McCrea, E. W. Mielke and Y. Ne'eman, Metric affine gauge theory of gravity: Field equations, Noether identities, world spinors, and breaking of dilation invariance, Phys. Rept. 258, 1 (1995).

[10] T. Dereli, M. Önder, J. Schray, R. W. Tucker and C. Wang, Non-Riemannian gravity and the Einstein-Proca system, Class. Quant. Grav. 13, L103 (1996) [arXiv:gr-qc/9604039].

[11] D. Diakonov, A. G. Tumanov and A. A. Vladimirov, Low-energy General Relativity with torsion: a systematic derivative expansion, [arXiv:1104.2432].

[12] P. Baekler and F. W. Hehl, Beyond Einstein-Cartan gravity: Quadratic torsion and curvature invariants with even and odd parity including all boundary terms, Class. Quant. Grav. 28, 215017 (2011) [arXiv:1105.3504].

[13] A. C. Hearn, REDUCE User's Manual, Version 3.5 RAND Publication CP78 (Rev. 1993).

[14] E. Schrüfer, EXCALC: A System for Doing Calculations in the Calculus of Modern Differential Geometry, (GMD-SCAI, 53757 St. Augustin, Germany, 1994).

[15] H. T. Nieh and M. L. Yan, An Identity in Riemann-Cartan Geometry, J. Math. Phys. 23, 373 (1982).

[16] H. T. Nieh, A Torsional Topological Invariant, Int. J. Mod. Phys. A 22, 5237 (2007).

[17] M. Adak, T. Dereli and L. H. Ryder, Dirac equation in spacetimes with torsion and nonmetricity, Int. J. Mod. Phys. D 12, 145 (2003) [arXiv:gr-qc/0208042].

[18] M. Adak, Non-Riemannian Theories of Gravitation and the Dirac Equation: Physical aspects of non-Riemannian extensions, (Lambert Academic Pub., Saarbrücken, 2010).

[19] J. D. Bjorken and S. D. Drell, Relativistic Quantum Mechanics (McGraw-Hill, New York, 1964).

[20] F. W. Hehl and Yu. N. Obukhov, Foundations of Classical Electrodynamics - Charge, flux, and metric (Birkhäuser, Boston, MA, 2003).

[21] M. Adak, T. Dereli and L. H. Ryder, Neutrino oscillations induced by spacetime torsion, Class. Quant. Grav. 18, 1503 (2001) [arXiv:gr-qc/0103046].

[22] F. W. Hehl, A. Macias, E. W. Mielke and Yu. N. Obukhov, On the structure of the energymomentum and the spin currents in Dirac's electron theory. In: On Einstein's Path. Essays in Honor of Engelbert Schucking, A. Harvey, ed. (Springer, NY, 1999) [arXiv:gr-qc/9706009].

[23] I. Kirsch, L. H. Ryder and F. W. Hehl, The Gordon decompositions of the inertial currents of 
the Dirac electron correspond to a Foldy-Wouthuysen transformation, [arXiv:hep-th/0102102].

[24] F. W. Hehl, P. von der Heyde, G. D. Kerlick and J. M. Nester, General Relativity with Spin and Torsion: Foundations and Prospects, Rev. Mod. Phys. 48, 393 (1976).

[25] H. Chen, F. H. Ho, J. M. Nester, C. H. Wang and H. J. Yo, Cosmological dynamics with propagating Lorentz connection modes of spin zero, JCAP 0910, 027 (2009) [arXiv:0908.3323].

[26] C. Lammerzahl, Constraints on space-time torsion from Hughes-Drever experiments, Phys. Lett. A 228, 223 (1997) [arXiv:gr-qc/9704047].

[27] V. A. Kostelecky, N. Russell and J. D. Tasson, Constraints on Torsion from Bounds on Lorentz Violation, Phys. Rev. Lett. 100, 111102 (2008) [arXiv:0712.4393].

[28] B. Gonçalves, Y. N. Obukhov and I. L. Shapiro, Exact Foldy-Wouthuysen transformation for a Dirac spinor in torsion and other CPT and Lorentz violating backgrounds, Phys. Rev. D 80, 125034 (2009), [arXiv:0908.0437]. 